\title{
La subordinación ideológica del saber médico andino en la salud intercultural chilena
}

\author{
Carlos Daniel Piñones Rivera \\ Universidad Arturo Prat, Iquique, Chile. \\ Email: carlospinonesrivera@gmail.com \\ Nanette Paz Liberona Concha \\ Universidad Arturo Prat, Iquique, Chile. \\ Email: nliberonac@gmail.com \\ Barbara Montecino Quenaya \\ Cooperativa de Trabajo Apacheta, Iquique, Chile. \\ Email: barbaraquenaya@gmail.com
}

\begin{abstract}
Resumen: Existen pocos estudios que aborden los procesos ideológicos presentes en las relaciones interculturales propiciadas por las iniciativas de Salud de los Pueblos Indígenas en Chile. Nuestro objetivo es mostrar cómo los elementos ideológicos del Saber Biomédico contribuyen a la subordinación del Saber Andino al interior de dichos programas. Para esto, expondremos material etnográficoproducido entre 2011 y 2012 en la comunidad de Camiña,Tarapacá. Posicionados desde la Antropología Médica Crítica, analizamos la ideología biomédica sobre el Saber Andino, así como las propuestas para la interculturalidad que surgen desde el personal biomédico. Concluimos reflexionandocómo la negación del carácter ideológico de la racionalidad biomédica, refuerza la subordinación del Saber Andino, produciendo un efecto opuesto al mentado en la política pública.
\end{abstract}

Palabras clave: Pluralismo médico; salud intercultural; salud pueblos indígenas; medicina tradicional.

\section{The ideological subordination of the Andean Medical Knowledge in Chilean Intercultural Health}

\begin{abstract}
There are few studies that address the ideological processes present in the intercultural relations fostered by the Indigenous Peoples Health initiatives in Chile. Our objective is to show how the ideological elements of Biomedical Knowledge contribute to the subordination of Andean Knowledge within these programs. For this, we will exhibit ethnographic material produced between 2011 and 2012 in the community of Camiña, Tarapacá. Positioned from the Critical Medical Anthropology, we analyze the biomedical ideology on the Andean Knowledge, as well as the proposals for the interculturality that arise from the biomedical personnel. We conclude by reflecting on how the denial of the ideological character of biomedical rationality reinforces the subordination of Andean Knowledge, producing an adverse effect on public policy.
\end{abstract}

Keywords: Medical pluralism; intercultural health; indigenous people health; traditional medicine.

\section{A subordinação ideológica do saber médico andino na saúde intercultural chilena}

Resumo: Existem poucos estudos que abordem os processos ideológicos presentes nas relações interculturais promovidas pelas iniciativas de Saúde dos Povos Indígenas no Chile. Nosso objetivo é mostrar como os elementos ideológicos do saber biomédico contribuem para a subordinação do Saber Andino dentro desses programas. Para isso, vamos expor material etnográfico produzido entre 2011 e 2012 na comunidade de Camiña, Tarapacá. Posicionada a partir da 
Antropologia Médica Crítica, analisamos a ideologia biomédica do Saber Andino, bem como as propostas de interculturalidade que surgem desde o pessoal biomédico. Concluímos refletindo como a negação do caráter ideológico da racionalidade biomédica reforça a subordinação do Saber Andino, produzindo um efeito contrário ao mencionado na política pública.

Palavras-chave: Pluralismo médico; saúde intercultural; saúde povos indígenas; medicina tradicional.

\section{Introducción}

Los perfiles epidemiológicos de todo el mundo muestran que uno de los grupos principalmente afectados por la inequidad en saludes el de los Pueblos Originarios (Gracey y King, 2009). En virtud de esto, la comunidad internacional realiza iniciativas fuertemente impulsadas por la Resolución "Salud de los Pueblos Indígenas” de la OMS(OPS, 1993). El año 1996 se crea en Chile el Programa Especial de Salud Pueblos Indígenas (PESPI), ${ }^{1}$ con el fin de disminuir estas brechas, y el 2003 ve la luz la Política de Salud Pueblos Indígenas,en vigor. A pesar de que esta política establece la interculturalidad como uno de sus principales ejes organizadores, presentando un imaginario de horizontalidad(Piñones Rivera, Mansilla Agüero, y Arancibia Campos, 2017)que guía las relaciones entre los saberes médicos de los Pueblos Originarios y el Saber Biomédico, nos es patente que la salud intercultural ${ }^{2}$ en Chile constituye un espacio de reproducción de relaciones de Hegemonía/Subalternidad.

El análisis que sigue apoya esta tesis, basándose en una investigación etnográfica cuyo campo se realizó entre los años 2011 y 2012, que buscó comprender los procesos de articulación entre los distintos saberes médicos coexistentes como parte del pluralismo médico ${ }^{3}$ propio de una comunidad aymara en el territorio chileno(Piñones Rivera, 2015). Estudiamos estos procesos desde el marco de la Antropología Médica Crítica (AMC), buscando comprender cómo participaban de la producción de relaciones de Hegemonía/ Subalternidad.Respecto de las relaciones entre el Saber Biomédico y el Saber Médico Andino, ${ }^{4}$ nuestro análisis pudo identificar dos tipos de procesos genéricos: los burocráticos y los ideológicos. Este artículo se concentrará en estos últimos, y mostrará cómo los elementos ideológicos del Saber Biomédico contribuyen a la subordinación del Saber Andino al interior de las iniciativas desplegadas por el PESPI en la Región de Tarapacá. Para esto, expondremos material tomado de la experiencia llamada "Casa del Kollire", en su interrelación con el dispositivo de atención primaria de la comunidad de Camiña, Región de Tarapacá.El valor de Camiña para pensar en estos procesos, radica en que ahí existe la más alta concentración nacional de agentes de salud andinos agrupados en una asociación indígena, y constituye una de las experiencias de más larga data en los programas de salud intercultural desarrollados bajo el alero de la Política de Salud Pueblos Indígenas,realizados con el pueblo aymara en Chile(Piñones Rivera, 2015).

\section{Metodología}

El material de campo en el cual se basa nuestro análisis es fruto del trabajo etnográfico realizado con los agricultores aymaras de Camiña entre 2011 y 2012. Estos datos forman parte de una investigación mayor(Piñones Rivera, 2015),cuyo diseño metodológico se estructuró específicamente para conocer los distintos saberes médicos coexistentes. Utilizamos la observación participante como técnica principal de recolección de información. Para obtener información sobre el Saber Andino se realizaron observaciones en el centro de atención "Casa del Kollire” (co-administrado por la Asociación Kollire y la Municipalidad), así como también en los hogares de algunos qullirinaja, yatirinaja ${ }^{5}$ o pacientes donde se realizaban las atenciones. Para el Saber Pentecostal se asistió a las sesiones de culto y a las concentraciones pentecostales realizadas en la iglesia local, y se visitaron las casas de personas enfermas para observar las vigilias. En el caso del Saber Biomédico las observaciones se llevaron a cabo en la posta de atención rural. Finalmente, para conocer el Saber de Autoatención se observó la convivencia cotidiana en los espacios domésticos. Los tópicos claves abordados fueron las prácticas de atención a los problemas de salud. De manera complementaria se realizaron 68 entrevistas a representantes de los distintos saberes médicos, cuyos tópicos centrales fueron las diversas 
enfermedades sufridas y conocidas, poniendo especial atención en sus procesos de desarrollo, causas y prácticas de atención.

Así, mientras de la observación participante obteníamos información directa sobre las prácticas de atención correspondientes a cada saber, las entrevistas nos entregaban información de las representaciones asociadas. Con esto generamos una interpretación de los elementos ideológicos característicos de los saberes médicos, así como de los procesos de articulaciones que se dan entre ellos. La información fue sometida a un análisis comparativo inicial de las distintas características ideológicas de los saberes médicos.

Para producir un análisis de los procesos de subordinación del Saber Andino, nos basamos en ambas técnicas realizadas.En lo que sigue damos especial énfasis a las entrevistas realizadas al personal biomédico, para mostrar los elementos que participan de la subordinación ideológica que nos interesa visibilizar. ${ }^{6}$

\section{Los saberes médicos como producciones ideológicas}

Siguiendo los supuestos de la AMC de Eduardo Menéndez,concebimos los saberes médicos como producciones ideológicas. Constituyen aquellas instancias desde las cuales se significa y actúa respecto de la salud, la enfermedad, la vida, la muerte, lo normal, lo desviado y todo aquello que eventualmente pueda incluirse dentro del Proceso Salud/Enfermedad/Atención(PSEA) (Menéndez, 1994). Son producciones de los conjuntos sociales, por lo que no se reducen a aquellos saberes producidos por los actores especializados (curadores, especialistas, profesionales de la salud, etc.)(Menéndez, 1994). Los saberes médicos cumplen una función terapéutica y varias no-terapéuticas: participan de la construcción del sentido común,de la subjetividad, delcontrol social y de la normatización. Estas últimas funciones son opacadas por la función terapéutica que es puesta en primer plano como respuesta a las demandas de atención que emergen desde los conjuntos sociales. No deben ser entendidos como entidades sino como procesos de transformación(Menéndez, 1996), consistiendo en una incesante estructuración de representaciones y prácticas en el espacio ideológico y al interior de Relaciones de Hegemonía/Subalternidad. Por todos estos elementos y funciones, los saberes médicos son parte importante del juego histórico, al participar del posicionamiento de los grupos sociales en dichas Relaciones de Hegemonía/ Subalternidad.

De acuerdo a nuestra concepción, el carácter ideológico de los saberes médicos no viene dado por su dimensión representacional, pues uno de sus elementos centrales son las prácticas que permiten la reproducción de las estructuras sociales.Tampoco por una supuesta falsa conciencia, concepción que los planteamientos gramscianos superan(Hall, 2010). Más bien viene dado porque todo saber médico es un "terreno en el que determinados grupos sociales toman conciencia de su propio ser social, de su propia fuerza, de sus propias obligaciones, de su propio devenir”(Gramsci, 1999, p. 201). Como plantea Hall, “El problema de la ideología está especialmente relacionado con los conceptos y los lenguajes del pensamiento práctico que estabilizan una forma particular de podery dominación”(Hall, 2010, pp. 134-135).Son dichos conceptos y lenguajes del pensamiento práctico biomédico local los que analizaremos a continuación.

\section{Elementos ideológicos participantes en las relaciones de subordinación del Saber Médico Andino}

\section{La ideología sobre sus efectos}

¿Cómo conciben los funcionarios biomédicos el quehacer de sus pares especialistas del Saber Andino? ¿Cuáles son los contenidos ideológicos que descubrimos en dichas concepciones? Partiremos con un fragmento de la entrevista a una profesionalquien, ante la pregunta sobre el trabajo del qulliri, con quien trabaja de manera coordinada en el marco del PESPI, responde:

$\mathrm{K}:$ "[...] en lo que es doloresmuscularessencillospuedeayudar[...] pero en el restode las cosas la verdades comobiendudosoparamí”. "C: ¿Dudoso en cuanto a qué? [...] K.: No, en realidad a que cumpla el 
efecto que requiere. De que la terapia de ellos haga un efecto que sea necesario para la persona. Por ejemplo, si yo voy a hacer una terapia yo quiero que el paciente vuelva a hacer sus actividades normales, que se sienta bien ¿cierto? [...] Pero ellos no, andan fajados enteros, con dolores, con parches, andan con la piel toda delicada porque esos parches se pegan y son súper firmes... [...] y en realidad no cumplen la función que debieran”. [Énfasis nuestro].

El primer contenido ideológico que encontramos es una valoración negativa respecto de la eficacia terapéutica del qulliri. Desde su punto de vista, solo podría ser eficaz en los casos en que se trata de dolores musculares sencillos, sin embargo, el trabajo del qulliriincluye una amplia gama de prácticas para tratar quebraduras, tronchaduras, mal aire, abertura de carne, empachos, etc. Es decir, un espectro que, si quisiéramos buscar un análogo biomédico, abarcaría las fracturas, luxaciones, parálisis faciales, afecciones gastrointestinales, etc.(Piñones Rivera, 2015). El supuesto biomédico es que el Saber Andino no puede, en principio, ser eficaz: "no hace el efecto necesario", no "cumple la función”. Mientras el biomédico aspiraría a que la persona "vuelva a hacer sus actividades normales, que se sienta bien", la aspiración del especialista andino no coincidiría con ellas.

¿De dónde proviene esta valoración de los efectos de las prácticas propias del Saber Andino? No lo hace de un estudio y análisis sistemático de las prácticas de los qullirinaja, pues ninguno de los funcionarios había realizado una investigación tal. En la literatura, este tipo de juicios ha sido expuesto como parte de los "valores ocultos" (Kirmayer, 1988), que operan como "supuestos tenaces de la biomedicina” (Gordon, 1988), uno de los cuales es el supuesto de la ineficaciade los saberes no científicos. Según Tambiah el juicio a priori de que la ineficacia es un atributo connatural de saberes concebidos como mágicos o religiosos, ${ }^{7}$ y que la ciencia es el dominio exclusivo en que tiene lugar la eficacia, se reconduce históricamente a la contribución del protestantismo a la mirada moderna, al calificar a la magia como falsa religión y como acción ineficaz, en tanto dios no se podría manipular(Tambiah, 1990, pp. 19-20).

La discusión contemporánea sobre la eficacia de los saberes médicos indígenas, ha puesto de relevancia que su complejidad no puede ser tratada adecuadamente en base a dichos juicios apriorísticos, cuestionando que el problema se pueda zanjar de manera unilateral desde la tradición del saber científico occidental(Waldram, 2000). Así, si desde el punto de vista biomédico el problema de la eficacia parece resolverse sometiendo cualquier práctica al ensayo clínico controlado, desde un punto de vista crítico este se desdobla en una serie de preguntas que develan su carácter político: ¿qué se define por eficaz?, ¿quién define qué es lo eficaz?, ¿cuáles son los criterios utilizados para dicha definición?(Waldram, 2000). Waldram muestra como la utilización de otros criterios da resultados favorables a la medicina llamada "tradicional", la que muestra ser bastante eficaz respecto de la resolución de problemas comunitarios, de la producción de sentidos culturalmente pertinentes, o de la relación conel territorio;todos aspectos que no son meros añadidos a los problemas de salud pues forman parte de las determinaciones psicológicas, sociales, culturales y económico-políticas de los mismos(Waldram, 2000).

Si recogemos los aportes de la actual discusión, parece sensato abordar el problema de la eficacia desde un concepto no estático, ni restringido a una única mirada (la biomédica).Más aún en contextos de pluralismo médico(Waldram, 2000, p. 615), donde los estudios de la AMC han analizado la conformación de relaciones de Hegemonía/Subalternidad concluyendo que el estatus de saber rechazado descansa más en cuestiones de poder que en estándares de verdad y eficacia (Waldram, 2000, p. 615).

Encontramos además el juicio simultáneo de iatrogenia. En el contexto de una política orientada por el principio de la interculturalidad, esto difícilmente se expresa de manera abierta y directa. No obstante, está presente en diversas magnitudes en todas las subespecialidades biomédicas con las que trabajamos. Un profesional señala:

“K: [...] en los casos más graves yo noto la diferencia que sí se producen retrocesos cuando van allá [donde el qulliri]. [...] K: Por las ventosas [...] porque es un efecto de una absorción de aire que hacen. [...] Entonces queda el círculo marcado y morado [...] y aparte los parches, que [...] comienzan a irritar la piel, entonces empiezan a producir heridas. Al final provoca más dolor todavía, y cuando llegan donde 
mí tengo que empezar desde cero, porque tengo que esperar que la herida sane, que baje el dolor y después tratarlo”.

Así las atenciones del qullirino solo son ineficaces, producen múltiples efectos negativos: irritaciones a la piel, heridas, marcas, aumento del dolor. Según otro profesional, las prácticas propias del qulliri pueden llegar al extremo de constituir conductas "aberrantes".

"E.: [...] Yo te digo cuando pasan cosas aberrantes... [...] echarme caca en una herida, ¿ya?... el tema cultural deja de ser aceptable... ¿ ¿me entiendes?, entonces uno interviene [...] a ver una vez escuché para quitar los cólicos, era quemar hojas dentro de la casa y ahí esa es una conducta aberrante; entonces ahí tú de la mejor forma intervienes. 'Señora ya, estoy de acuerdo con las cosas tradicionales, pero piense que si yo quemo cosas al interior de la casa puedo quemar las cosas, puedo quemar al bebé, el humo también puede quemar todo lo que es el conducto respiratorio y etc.'. Y ahí voy explicando y dando todas las condiciones del por qué no es adecuado"

Como se observa, se entiende que las prácticas basadas en el Saber Andino pueden exponer a daños a las personas, en cuyo caso sus acciones ("el tema cultural") dejan de ser aceptables, exigiendo la intervención del personal biomédico para evitar o contrarrestar dicho perjuicio.Lo que nos interesa mostrar es que, en la línea del análisis de las relaciones de Hegemonía/Subalternidad entre los saberes médicos, el Saber Biomédico despliega una vigilancia de la iatrogenia del Saber Andino, pues la iatrogenia dibuja el límite implícito de su tolerancia. La existencia de este límite refleja una dinámica asimétrica en el ejercicio del poder, pues en las entrevistas al personal biomédico no se planteó nunca el problema de la "tolerancia respecto del Saber Biomédico”, por mucho que sus prácticas o la lógica que lo estructura genere también situaciones iatrogénicas, según constatan algunos relatos de los pacientes(Piñones Rivera, 2015) y refleja copiosa literatura contemporánea(Conrad, 2007; Gøtzsche, 2014; Jamoulle, 2015; Moynihan, Doust, y Henry, 2012; Welch, Schwartz, y Woloshin, 2011).

En términos prácticos, la ausencia de una problematización de la distribución de la iatrogenia entre los saberes médicos, produce una consecuencia negativa para los propios objetivos de interculturalidad del PESPI,pues funda una reticencia en el equipo profesional a derivar personas al Saber Andino:

“K: [...] entonces como te comentaba el otro día, me costó el hecho de yo poder derivar pacientes, yo me negaba a que vayan allá. Entonces ellos me decían '¿puedo ir al médico andino?’ No, yo quiero ver mis resultados y después vas donde él si quieres, si no te resulta conmigo... y se iban, los perdía. Entonces tengo que verlos paralelamente pero yo veo cuando van al médico andino y yo noto un retroceso cuando llegan conmigo de nuevo".

Así, el problema de la derivación no se relaciona solo con el orden de las representaciones de los actores, pues está obstaculizado por una subordinación burocrática que incluye la insuficiencia en el desarrollo de protocolos, así como la lógica jerárquica de las responsabilidades propia del estatuto administrativo, que hace recaer la responsabilidad de cualquier problema en el proceso de atención a la autoridad biomédica, única legitimada por el Estado chileno(Piñones Rivera, 2015).

Como se descubre en la cita, dicha subordinación burocráticase apuntala y refuerza por la subordinación ideológica que valora el Saber Andino como fundamentalmente iatrogénico(Piñones Rivera, 2015). En efecto, la sumatoria del descrédito de la eficacia y la identificación del Saber Andino como iatrogénico, nos muestra que este es producido como no-saber. Exploraremos esto en el siguiente apartado.

\section{La ideología sobre el saber}

¿A que nos referimos por producción del Saber Andino como no-saber? A que desde los supuestos biomédicos no solo existe una valoración negativa de la eficacia o del potencial curador de sus intervenciones. El problema yacería en las características propias de este saber, el que solo podría ofrecer una mirada superficial o externa respecto de los procesos de enfermedad en cuestión: 
"K.: Es que yo no estoy muy de acuerdo la verdad [...] obviamente porque yo tengo estudios y sé que hay una alteración fisiológica, que hay una alteración como más orgánica se puede decir, y ellos lo ven como todo por fuera, entonces con ellos con un masaje con lo que sea, no consideran el peso, ni la actividad física y esas son cosas que si influyen bastante en cualquier dolor [...] Entonces pasan por alto muchas cosas. Una fractura ellos llegan y arreglan el hueso y lo entran y no ven qué estructuras están dañadas, que pasó a llevar un nervio, que pasó a llevar un músculo... bueno antiguamente era así, se quedaban así y no había otro remedio y ahora con la medicina sí se pueden corregir, si se pueden evitar que se produzcan otras lesiones”[énfasis nuestros]

En general el personal biomédico entrevistado comparte el supuesto de que el Saber Andino constituye una mirada "por fuera", que "pasa por alto muchas cosas", mientras el Saber Biomédico sería un conocimiento profundo y detallado, pues permitiría conocer lo “orgánico” y su fisiología,y de esta manera las estructuras que están dañadas.

Como han mostrado varios autores (De la Cadena, 2015; Good, 2008; Martínez-Hernáez, 2011; Tambiah, 1990), a la base de estasdescalificacionesestá la distinción histórica entre ciencia y creencia, según la cual todo saber no científico se basaen meras creencias, en tanto desconoce las causas naturales. La distinción se encuentra presente en los inicios de la biomedicina, pues es parte del acervo heredado del corpus hipocraticum, siendo "La enfermedad sagrada" el primer texto en que se documenta la identificación de toda explicación nonaturalista como una forma de ignorancia (Tambiah, 1990, pp. 9-10). Desde ahí ha llegado a constituir parte del etnocentrismo occidental según refleja elocuentemente Latour: "Nosotros los occidentales no podemos ser una cultura entre otras porque también movilizamos la naturaleza. No, como lo hacen las otras sociedades, una imagen o una representación simbólica de la naturaleza, sino la naturaleza tal y como es, o por lo menos tal y como las ciencias la conocen” (Latour, 2007, p. 145).

Otro elemento descalificatorio es que, en la lógica racional moderna, para llegar a detentar legítimamente un saber sobre las causas naturales, es preciso haber realizado un recorrido validado por una institucionalidad formal de educación. Cuestión beneficiosa, siempre y cuando no excluya las formas propias de legitimación de comunidades y pueblos originarios.En nuestra cita, mientras el biomédico "tiene estudios" (formales), el qulliri carente de ellos, estaría condenado a una mirada superficial sobre los procesos de salud. Un supuesto ideológico importante es que los estudios formales garantizarían un acceso a una forma más profunda y detallada sobre cualquier aspecto del PSEA, siendo el núcleo de esa garantía el conocimiento según la llave de bóveda que conferiría el código orgánico-fisiológico.No obstante, se ha planteado que, sobre una serie de dimensiones del PSEA, el Saber Biomédico se muestra especialmente impotente debido a su determinismo biológico (MartínezHernáez, 2008).

En todos estos casos, constatamos lo que Boaventura de Sousa Santos ha denominado la imposición de una monocultura del saber, consistente "en la transformación de la ciencia moderna y de la alta cultura en [...] cánones exclusivos de producción de conocimiento” (2009, p. 110). Uno de los efectos de dicha imposición es que "todo lo que el canon no legitima o reconoce es declarado inexistente. La no existencia asume aquí la forma de ignorancia o de incultura”(De Sousa Santos, 2009, p. 110).

Articulando los dos grandes bloques desarrollados hasta ahora, podemos evidenciar un procesoideológico de subordinación guiado por una racionalidad que podríamos parafrasear así: existe una valoración negativa del Saber Andino cuando se refiere a sus efectos, que los sitúaen un continuum que va de la ineficacia a la iatrogenia.La valoración negativa se funda en el juicio de "superficialidad" del Saber Andino, que es producido como forma de ignorancia al evaluarse desde el parámetro monocultural del discurso naturalista, establecido como único canon legítimo.

\section{La ideología sobre lo inter}

El proceso de producción del Saber Andino como no-saber halla un correlato claro en el tipo de propuestas que los profesionales biomédicos esbozan cuando piensan los pasos futuros que debería dar la interculturalidad. 


\section{Capacitar}

Una de las principales propuestas apunta a rectificar la falta de saber diagnosticada en el Saber Andino a través de procesos de capacitación hacia sus agentes.

“C:¿hacia dónde crees tú que debería avanzar la interculturalidad en salud acá en Camiña? K.: Como te decía creo que capacitándolos a ellos [...] yo creo que deberían [...] enseñarles algo por lo menos, no los fundamentos de por qué se hacen, sino de cómo deberían hacerse las maniobras, y con eso trabajar más en conjunto, yo creo que tendríamos mucha más confianza de los dos lados”

En este tipo de argumentos se descubre una fuerte producción del saber como inexistente, pues muy seriamente se planteaba que habría que enseñarle a los qullirinaja los rudimentos de su quehacer, por ejemplo, un masaje:

$\mathrm{K}$ : “entonces si uno no sabe hacer un masaje bien, porque yo he ido a ver los masajes que hace [el qulliri], y hay direcciones donde tú das masajes; entonces si tu das un masaje hacia un lado estas activando la musculatura y hacia este otro lado la estás relajando. Entonces llegan todo contracturados... entonces de repente, quizás enseñarle para que no pierdan eso, porque yo encuentro que está bien que ellos tengan eso" [oficio o trabajo]

Lo mismo pasa con las parteras, a quienes habría que enseñarles qué es un embarazo:

“K: [...] el año pasado venían médicos y los capacitaban. Capacitaban a las parteras, capacitaban a los yatiris o los qulliris. Los capacitaban para que en realidad supieran un poco más también, por ejemplo, cuando las parteras dicen que las dan vueltas, las cuelgan de los pies, les ponen una faja, entonces las capacitaron en relación a lo que es un embarazo. Para que no tenga problemas y no sufra algún problema del embarazo con las técnicas que tengan ellos”

En ambos casos el potencial subordinante dentro del discurso biomédico puede llegar a suponerun desconocimiento de las experiencias elementales de un saber médico específico. En este contexto nos parece legítimo interrogar: ¿qué sentido tiene que una partera practique las maniobras propias del Saber Biomédico? O ¿qué podría enseñar un especialista biomédico sobre las maniobras que son propias del Saber Andino?Así, las iniciativas de articulación producen una transformación de las prácticas(Piñones Rivera, 2015), conduciendo a una "biomedicalización de la medicina tradicional” (Poltorak, 2013; Tantipidoke, 2017; Waldram, 2000). En esta línea, se ha identificado a la alienación como uno de los principales peligros de las relaciones supuestamente interculturales, que ocurre cuando "un grupo (o miembros de él) se asimila y deja absorber en otro grupo más poderoso con pérdida de la propia identidad” (Albó, 2011).

Lo que nuestro material descubre es una dificultad generalizada de la biomedicina para reflexionar respecto de sus propios supuestos culturales, apuntalada en la asunción que “cultura” es un descriptor de las experiencias de los pacientes o de los otros saberes, y no de los agentes especializados biomédicos ni de su saber. La biomedicina sería una “cultura de la no cultura” (Taylor, 2003).

Asumir a la biomedicina como tal, permite comprender por qué en general las capacitaciones conducen a asimilar las prácticas de los especialistas médicos a aquellas de los biomédicos, definiendo una posición subalterna:no se percibe como un problema de relación entre dos o más culturas, sino como la relación entre un saber y una creencia cultural. Permite comprender también por qué muchas veces la capacitación, al dar ciertos rudimentos de la cultura indígena al personal biomédico, fortalece el Saber Biomédico a través de un “suplemento sociocultural”(Piñones Rivera et al., 2017), sin fortalecer el Saber Andino y sus agentes. Y es que en contextos de subalternización histórica, el fortalecimiento del Saber Médico de los Pueblos Originarios, debe conllevar transformaciones del currículum oculto(Becker, Geer, Hughes, y Strauss, 1992), de la estructura burocrática(Piñones Rivera, 2015), de la definición del presupuesto en salud (Toledo Llancaqueo, 2007), así como de cuestiones de índole estructural como el reconocimiento constitucional de los pueblos originarios (y no solo de sus “Sistemas Culturales de Salud”) o el respeto del derecho a la autodeterminación. 


\section{Controlar}

Siendo este el diagnóstico que realiza el personal biomédico del saber de sus pares en el terreno ideológico de la interculturalidad, ¿cuál es entonces el espacio de legitimidad y validez que confiere al Saber Andino? El saber del qulliri es considerado legítimo y útil principalmente en los siguientes casos:

a) Casos de origen psicológico:

“C: [...] Tú me decías que observabas un retroceso, ¿eso tú en la generalidad de los casos tú lo ves que es así? K.: No siempre, la gente la verdad es que es bastante hipocondriaca,[...] entonces tú con la evaluación sabes cuán grave es o cuán no, entonces yo lo veo y no... En realidad es más chiva[embuste]que lo que tiene, entonces de repente van y se les pasa de inmediato”.

b) Casos simples:

"K.: El qulliri lo que hace más es un refuerzo de... no sé... como un masajeador, como que fortalece y a través de las hierbas también produce reacciones positivas, pero cosas más complejas, como una fractura por ejemplo, no lo veo con las herramientas"

c) Casos falsos, es decir, aquellos inventados con alguna finalidad ganancial como podrían ser los casos en que el estado de enfermedad produce una excepción de ciertas obligaciones.

d) Casos de oposición al tratamiento biomédico por apego a las “tradiciones” andinas.

"C: ¿En qué caso tu derivas los pacientes al qulliri? K.: [...] De repente cuando veo que no cede por ejemplo o son muy porfiados [...]no van a seguir mi tratamiento[...] yo los derivo para allá y yo sé que psicológicamente ellos saben que con una sesión, con una ventosa o con un masaje o con un estiramiento del nervio ellos van a quedar bien”.

Como se observa, la mayoría de estos casos caben dentro de la categoría de lo imaginario y sólo una es concebida como poseedora de eficacia real (casos simples), pero perfectamente prescindible. La producción de dicho espacio restringido de legitimidad para el qulliri constituye una de las formas de control de este saber y muestra que la interculturalidad en salud puede ser un buen ejemplo de campo social en el cual se legitime solo a los indios permitidos(Hale, 2004). Aquí el indio permitido sería aquel que se mantiene en el restringido espacio de validez y legitimidad definido por el Saber Biomédico.

\section{Traducir}

No todas las propuestas para la interculturalidad descansan en una valoración negativa del quehacer de los especialistas andinos. En el discurso de los profesionales encontramos enunciados que valoran ciertos aspectos:

"E: Claro, porque recetas caseras o las mismas hierbas, mates... [...] si tú lo piensas estoy con unresfrío común estacional y se me aconsejó, o milenariamente yo sé, que tengo que tomar mate. Nosotros ¿qué decimos desde lo occidental? Aumente la ingesta de líquido. Porque uno se deshidrata y humidifica las secreciones, salen y la mucosa que esta irritada la protejo. Nosotros decimos líquidos y la manera tradicional es mate. Por eso digo yo, hay una parte científica que nosotros avalamos, que no impedimos”.

Un supuesto ideológico de esta racionalidad es que todos los saberes médicos son traducibles al lenguaje y concepción biomédica. Esto en virtud de un mínimo común, la parte aquí denominada "científica” del Saber Andino, que la biomedicina estaría en condiciones de sancionar y avalar como eficacia real en virtud de sus características ideológicas(Waldram, 2000). Parafraseando a De la Cadena (2015), dicho mínimo común sería la similitud onto-epistémicaque permitiríatraducir la diferencia en similitud.

Un elemento ideológico implícito es que es posible expoliar el Saber Andino de todo lo que tiene de “creencia”,depurando aquella esencia de eficacia, que sería resguardada de los velos de la ilusión al traducirla al lenguaje naturalista. Así, aquella porción rescatable del Saber Andino -no iatrogénica ni ineficaz, sino su 
“parte científica”-sería salvaguardada al extraerla del terreno de la proto-ciencia o la pseudo-ciencia, para alcanzar su lugar en la sistematización real que solo la ciencia podría realizar.

La traducción naturalista consistiría en expresar dichos fragmentos de verdad, a través de un lenguaje que busca excluir sistemáticamente tanto la parte que corresponde a la agencia humana como al azar, permitiendo rescatar el supuesto principio de su eficacia, que siempre correspondería al orden de las causas naturales(Rosset, 1974). Así liberada, la verdad del Saber Andino podría reconocer los principios de su eficacia al mirarlos en aquel "espejo de la naturaleza” que sería el lenguaje naturalista. Como ha mostrado Martínez-Hernáez, dicha pretensión es el resultado de una estrategia de ocultación o velamiento de la condición sociopolítica de la ciencia, que opera a través de los procedimientos de asocialización, universalización y neutralización(MartínezHernáez, 2011, p. 49).

Ante ella, importa establecer que no es claro que el lenguaje biomédico sea el más adecuado para traducir todas las experiencias a lo largo del globo, considerando la rica pluralidad de concepciones culturales referidas a la salud y la enfermedad(Good, 2008, p. 23). Más aún, la idea de que la ciencia tiene la facultad de establecer lo que es creencia de lo que no lo es, también ha sido cuestionada(Good, 2008, p. 22), reconociendo que la biomedicina es un sistema ideológico con sus propios supuestos onto-epistémicos que no son neutros(Lock y Gordon, 1988; Menéndez, 1983; 2005).

\section{Instrumentalizar}

Existe un tipo de utilidad que el Saber Andino presta ala biomedicina, y que no cabe dentro del reducido espacio de legitimidad que se da a los qullirinaja. Nos referimos a aquellos casos significados por el personal biomédico como "oposicionistas", una de las principales preocupaciones en su trabajo con Pueblos Originarios. En efecto, uno de los pocos procesos de su trabajo que el personal biomédico atribuye al ámbito de lo social es el de producir un vínculo que facilite la "adherencia al tratamiento". Aquí es donde se hace relevante lo que se ha denominado "negociación cultural”, que se manifiesta "cuando las creencias culturales y las expectativas de los pacientes secontraponenalasdelprofesionaldesalud interfiriendo en la confianza y adherencia al proceso terapéutico"(Alarcón, Vidal, y Neira Rozas, 2003, p. 1064).Pues se ha detectado una escasa adherencia a tratamientos farmacológicos cuando estos son considerados como opuestos al marco de creencias del paciente (Alarcón et al., 2003).

Notemos que la valoración que supone el uso del Saber Andino para conseguir la adherencia al tratamiento biomédico, puede perfectamente prescindir de una valoración positiva sobre su validez, veracidad y eficacia. Esto visibiliza el valor instrumental que la biomedicina confiere al Saber Andino, pero a la vez dibuja el límite de su valoración; pues esta se condiciona, cuando se percibe que los beneficios de la adherencia al tratamiento empiezan a ser contrarrestados o superados por el potencial iatrogénico atribuido.

"E: Y esas son las cosas que tu negocias[...] cuando tienen algún desgarro matan un perro negro y se ponen la parte del perro en el pecho, entonces tu respetas esas cosas porque es necesario si tú quieres un buen lazo, no puedes juzgar, eso y ahí es donde tú juegas, porque ¿qué es lo que te va a hacer a ti? Ya, mató el perro o las patas del llamo o guanaco, no va a interferir con algo severo. Pero si es una herida o una quemadura y la gente cree que echándose el guano de la cabra se le va a pasar, ahí uno debería intervenir, porqueno puedo echarme excremento en una quemadura. Pero con cosas que tú sabes que no va a dañar, respetas”[Énfasis nuestros]

Por último, existe otra utilidad del Saber Andino para el Saber Biomédico, pero que opera a nivel comunitario. Es el hecho de que, a través de su inclusión al interior del espacio de acción biomédico, las personas y la comunidad se sentirían "tomados en cuenta".

"C: ¿Que sería lo positivo y lo negativo del uso que la población hace de estos usos terapéuticos paralelos a los de la posta? B: Yo creo que más que nada sentirse integrado en cuanto a su cultura y su modo de ver las cosas en una cuestión que fue como impuesta, la cantidad de años que están acá y milenariamente están estas cuestiones, o sea nosotros nos metimos en su ambiente, no sólo nosotros, sino también cara- 
bineros [policías], etc. Más que nada sentirse integrados, sentirse como tomados en cuenta. Y también el que tengan un espacio formal es importante porque les sirve para aceptar el tema biomédico como tal porque dicen: 'está la casa del médico andino asociado, si bien no al lado, pero administrativamente relacionando con nosotros, será por algo, será porque ambas cosas nos ayudan’. Por un lado, nos sirve para que ellos sientan que son tomados en cuenta en cuanto a sus creencias y también para que ellos nos validen a nosotros, como también una opción, eso ha sido importante para que se haga esta transformación como de los algoritmos a seguir para llegar a una solución del problema de salud”.

\section{Conclusión}

El material aquí analizado nos permite constatar cómo la salud intercultural, lejos de ser un espacio para la horizontalidad, constituye un campo social en el cual la articulación de saberes médicos contribuye con la subordinación ideológica del Saber Andino. En la medida que los elementos ideológicos analizados se reafirman como "supuestos tenaces” y se niega el carácter ideológico-cultural de laracionalidad biomédica, se reproduce y refuerza la subordinación del Saber Andino, produciendo un efecto opuesto al que está mentado en la política pública, de fortalecimiento y salvaguarda.

La política de Salud Pueblos Indígenas está en una posición privilegiada para generar las condiciones que permitan cuestionar dichos supuestos tenaces. Pero a la vez, se encuentra en la paradójica posición de ser un instrumento de un Estado Monocultural, que históricamente ha legitimado el Saber Biomédico como guía para organizar su aproximación burocrática frente a los problemas de salud. Y cuya aproximación a la diversidad cultural, caracterizada como Multiculturalismo Neoliberal,desconoce los derechos colectivos y territoriales de los Pueblos Originarios, negando sistemáticamente su derecho a la autodeterminación(Díaz Polanco, 2007).

Parafraseando los términos en los cuales se ha elaborado esta política, nos parece que hasta que nose analicen y transformen sistemáticamente los supuestos tenaces de la biomedicina, en la estructura y contenidos, es muy difícil que el actual reconocimiento conduzca a la salvaguarda, y que la complementariedadconduzca al fortalecimiento de los saberes médicos de los Pueblos Originarios.

\section{Agradecimientos}

El presente artículo está basado en la ponencia presentada en el II Coloquio Internacional de Estudios sobre culturas Originarias de América, organizado por el el Programa de Estudios sobre Culturas Originarias de América de la Casa de las Américas, realizado en La Habana entre el 11 y 14 de Octubre del 2016. El autor principal agradece a Becas Chile para Doctorado en el Extranjero de ConicytChile por el financiamiento para esta investigación.

\section{Notas}

\footnotetext{
${ }^{1}$ El PESPI tiene como objetivo general "contribuir a la disminución de brechas de inequidad, en la situación de salud de los pueblos indígenas, a través de ia construcción participativa de planes de salud que reconozcan la diversidad cultural, promuevan la complementariedad entre sistemas médicos y provean servicios de salud adecuados que respondan a necesidades, derechos y perfiles epidemiológicos específicos”. Para esto sus objetivos específicos son: “1. Generar acciones que permitan contribuir a mejorar la calidad, accesibilidad y resolutividad en la atención de salud a través de estrategias de facilitadores, asesores, sensibilización y capacitación con enfoque intercultural; 2. Incorporar la transversalización del enfoque intercultural en los programas de salud mediante la ejecución de estrategias orientadas a mejorar la pertinencia cultural de las actividades de salud; 3. Apoyar procesos orientados al reconocimiento, salvaguarda, fortalecimiento y complementariedad de los sistemas culturales de salud de los pueblos indígenas” (MINSAL, 2011, p. 5).

${ }^{2}$ Para la política de salud pública chilena la interculturalidad es definida como "un proceso social interactivo, de reconocimiento, respeto, horizontalidad y colaboración entre dos o más culturas, en un espacio determinado. Interculturalidad significa entonces, la promoción de relaciones de confianza, reconocimiento mutuo, comunicación efectiva, cooperación y convivencia; y derecho a la diferencia” (MINSAL, 2006, p. 21). Posicionandonos críticamente, entendemos que dicha definición forma parte de la salud intercultural como terreno ideológico característico del multiculturalismo neoliberal, a través del cual el Estado aborda el problema de la inequidad en salud propia de los pueblos originarios, desde directrices ideológicas socioculturalistas, voluntaristas y ahistoricistas. Este terreno ideológico produce un "imaginario de la horizontalidad" en contextos de asimetría de poder, que opaca la participación que el modo de
} 
producción capitalista -y el Estado que lo instrumenta- tienen en la producción de la misma inequidad en salud. Para un análisis crítico detallado de la salud intercultural chilena Cfr. Piñones Rivera, Mansilla Agüero, y Arancibia Campos (2017). ${ }^{3}$ Según Perdiguero (2006) el pluralismo médico es “la existencia en una gran parte de las sociedades humanas de este mundo globalizado
de modos diversos de entender la salud y la enfermedad, de diagnosticar y tratar los padecimientos” (p. 33). Como puntualiza Eduardo
Menéndez, “domina en las sociedades actuales, dentro de los diferentes conjuntos sociales estratificados que las constituyen y más allá
de la situación de clase o de la situación étnica [...] en nuestras sociedades la mayoría de la población utiliza potencialmente varias
formas de atención no sólo para diferentes problemas, sino para un mismo problema de salud” (Menéndez, 2003, p. 186).

${ }^{4}$ En adelante Saber Andino.

${ }^{5}$ Qullirinaja es el plural de qulliri, que significa “persona con cualidad de curar” (García Choque, 2005). En nuestro campo se utilizaba para referirse a las personas que saben principalmente del uso de hierbas, emplastos o problemas de huesos y nervios. A diferencia, yatiri significa "el que sabe" (Layme, 2004) y su conocimiento especializado (si bien no restrictivo), es sobre los llamados "problemas espirituales”: susto, agarradura, mal impuesto, etc. (Piñones Rivera, 2015). Cfr. también Choque Mariño (2012).

\begin{abstract}
${ }^{6}$ Resulta importante señalar que, de acuerdo con nuestras entrevistas, en general el personal biomédico no contaba con formación especializada en salud intercultural o antropología médica, más allá de la capacitación inicial que varios recibieron de parte del Servicio de Salud Iquique, antes de integrarse en el equipo de salud de Camiña. No obstante, y según veremos desarrollado en los siguientes apartados, queda bastante en entredicho el supuesto de que las instancias de capacitación de por sí basten para contrarrestar los supuestos ideológicos de los currículums explícito y oculto de la formación de las ciencias de la salud en Chile. El carácter marginal que la Medicina Social, la Salud Colectiva y la Antropología Médica Crítica tienen en dichos currículums, puede ser un buen indicador de lo lejos que Chile está de alcanzar el estándar de Interculturalidad Crítica necesario para combatir, en el terreno ideológico de la salud, la inequidad que afecta a los pueblos originarios. A esto hay que añadir que toda interculturalidad es íntimamente contradictoria como política pública en el seno del neoliberalismo, que es en última instancia la fuente de producción de dicha inequidad, en tanto los procesos de acumulación vulneran directamente los derechos colectivos de los Pueblos Originarios (Díaz Polanco, 2007; Hale, 2004; Piñones Rivera, Mansilla Agüero, y Arancibia Campos, 2017).
\end{abstract}

${ }^{7}$ No compartimos la identificación del saber médico andino con la categoría de “magia” o "religión”, por el lastre colonial que suponen esas categorías (Asad, 1993; Tambiah, 1990). Para una discusión sobre la diferencia entre saber, sistema y modelo médico Cfr. Piñones Rivera (2015).

\title{
Bibliografía
}

Alarcón, A. M., Vidal, A., y Neira Rozas, J. (2003). Salud intercultural: elementos para la construcción de sus bases conceptuales. Revista Médica de Chile, (131), 1061-1065.

Albó, X. (2011). Interculturalidad y Salud. En Salud e Interculturalidad en América Latina. Perspectivas antropológicas (pp. 65-74). Quito, Ecuador: Abya-Yala.

Asad, T. (1993). Genealogies of religion: Discipline and reasons of power in Christianity and Islam. London, UK: The Johns Hopkins University Press.

Becker, H. S., Geer, B., Hughes, E. C., y Strauss, A. L. (1992). Boys in White. Sudent Culture in Medical School. Chicago, USA: The University of Chicago Press.

Choque Mariño, C. (2012). Fortunato Manzano, el último Yatiri.Arica, Chile: CONADI.

Conrad, P. (2007). The Medicalization of Society. On the Transformation of Human Conditions into Treatable Disorders. Baltimore, USA: The Johns Hopkins University Press.

De la Cadena, M. (2015). Earth beings: ecologies of practice across Andean worlds. Durham, USA: Duke University Press.

De Sousa Santos, B. (2009). Una epistemología del sur. Ciudad de México, México: Siglo XXI Editores.

Díaz Polanco, H. (2007). Elogio de la diversidad: globalización, multiculturalismo y etnofagia.Ciudad de México, México: Siglo XXI Editores. 
García Choque, P. A. (2005). Aru Pirwa. Diccionario Aymara-Castellano y Castellano-Aymara.(3a ed.). Iquique, Chile: Imprenta Graficolor.

Good, B. J. (2008). Medicine, Rationality, and Experience. Cambridge, USA: Cambridge University Press.

Gordon, D. (1988). Tenacious Assumptions in Western Medicine. In Biomedicine Examined. Dordrecht, Netherlands: Springer Netherlands.

Gøtzsche, P. C. (2014). Why i think antidepressants cause more harm than good. The Lancet Psychiatry, 1(2), 104106. https://doi.org/10.1016/S2215-0366(14)70280-9

Gracey, M., y King, M. (2009). Indigenous health part 1: determinants and disease patterns. The Lancet, 374(9683), 65-75. https://doi.org/10.1016/S0140-6736(09)60914-4

Gramsci, A. (1999). Cuadernos de la cárcel. Edición crítica del Instituto Gramsci a cargo de Valentino Gerratana. Tomo 4 (Vol. 1). Ciudad de México, México: Ediciones Era. https://doi.org/10.4324/9780203625521

Hale, C. R. (2004). Rethinking Indigenous Politics in the Era of the “ Indio Permitido “. NACLA Report on the Americas, 38, 16-21.

Hall, S. (2010). Sin garantías. Popayán, Colombia: Envión editores.

Jamoulle, M. (2015). Quaternary prevention, an answer of family doctors to overmedicalization. International Journal of Health Policy and Management, 4(2), 61-64. https://doi.org/10.15171/ijhpm.2015.24

Kirmayer, L. J. (1988). Mind and Body as Metaphors: Hidden Values in Biomedicine. In Biomedicine Examined. Dordrecht, Netherlands: Springer Netherlands.

Latour, B. (2007). Nunca fuimos modernos. Buenos Aires, Argentina: Siglo XXI Editores.

Layme, F. (2004). Diccionario bilingüe aymara-castellano. La Paz, Bolivia: Edición Consejo Educativo Aymara.

Lock, M., y Gordon, D. (1988). Biomedicine examined. (M. Lock y D. Gordon, Eds.). Dordrecht, Netherlands: Springer Netherlands.

Martínez-Hernáez, Á. (2008). Antropología Médica: Teorías sobre la cultura, el poder y la enfermedad. Barcelona, España: Anthropos.

Martínez-Hernáez, Á. (2011). La copia de los hechos. La biomedicina, el poder y sus encubrimientos. Quaderns de l'Institut Català d'Antropologia, 27, 45-64.

Menéndez, E. L. (1983). Hacia una práctica médica alternativa: Hegemonía y autoatención (gestión) en salud. Ciudad de México, México: Cuadernos de la Casa Chata. CIESAS.

Menéndez, E. L. (1994). La enfermedad y la curación ¿Qué es medicina tradicional? Alteridades, 4(7), 71-83.

Menéndez, E. L. (1996). El saber popular como proceso de transformación: tipos de articulación entre la biomedicina y la medicina popular. In J. A. González Alcantud y S. Rodríguez Becerra (Eds.), Creer y curar: la medicina popular (pp. 31-63). Granada, España:Diputación Provincial de Granada.

Menéndez, E. L. (2003). Modelos de atención de los padecimientos: de exclusiones teóricas y articulaciones prácticas. Ciencia \& Saúde Coletiva, 8(1), 185-207 
Menéndez, E. L. (2005). El Modelo Médico y la Salud de los Trabajadores. Salud Colectiva, 9-32.

MINSAL. (2006). Política de Salud y Pueblos Indígenas. Santiago, Chile: MINSAL.

MINSAL. (2011). Resolución Exenta $n^{\circ}$ 11, de 18 de enero de 2011. Aprueba Programa Especial de Salud y Pueblos Indígenas “PESPI”. Santiago, Chile: Minsal.

Moynihan, R., Doust, J., y Henry, D. (2012). Preventing overdiagnosis?: how to stop harming the healthy. British Medical Journal, 3502(May), 1-6. https://doi.org/10.1136/bmj.e3502

OPS. (1993). Resolución V. Salud de los Pueblos Indígenas. Ginebra, Suiza.

Perdiguero, E. (2006). Una reflexión sobre el pluralismo médico. En Salud e Interculturalidad en América Latina. Perspectivas antropológicas (pp. 33-49). Quito, Ecuador: Abya-Yala.

Piñones Rivera, C. (2015). La Mala Hora. Articulaciones en el pluralismo médico de agricultores precordilleranos aymaras chilenos. Universitat Rovira i Virgili. Retrieved from http://www.tdx.cat/handle/10803/320188

Piñones Rivera, C., Mansilla Agüero, M. Á., y Arancibia Campos, R. (2017). El imaginario de la horizontalidad como instrumento de subordinación: la Política de Salud pueblos indígenas en el multiculturalismo neoliberal chileno. Saude e Sociedade, 26(3), 751-763. https://doi.org/10.1590/S0104-12902017169802

Poltorak, M. (2013). The efficacy and self-efficacy of treatment: Ethnomedical aspirations, biomedical inhibitions, and health outcomes. Medical Anthropology Quarterly, 27(2), 272-291. https://doi.org/10.1111/maq.12027

Rosset, C. (1974). La Antinaturaleza. Madrid, España: Taurus.

Tambiah, S. J. (1990). Magic, science and religion and the scope of rationality. Cambridge, UK: Cambridge University Press.

Tantipidoke, Y. (2017). Local healing in northern Thailand: An anthropological study of its effectiveness. University of Amsterdam. https://doi.org/10.1177/1745691612459060.

Taylor, J. S. (2003). Confronting “Culture” in Medicine’s “Culture of No Culture.” Academic Medicine, 78(6), 555-559.

Toledo Llancaqueo, V. (2007). Presupuesto del Sector Público y Políticas Indígenas. Chile 1994- 2008.

Waldram, J. B. (2000). The efficacy of traditional medicine: current theoretical and methodological issues. Medical Anthropology Quarterly, 14(4), 603-625.

Welch, H., Schwartz, L., y Woloshin, S. (2011). Overdiagnosed: making people sick in the pursuit of health. Boston, USA: Beacon Press. 